\title{
GENERALIZATION OF MEIR-KEELER TYPE FIXED POINT THEOREMS
}

\author{
R. P. PANT, VYOMESH PANT AND V. P. PANDEY
}

\begin{abstract}
The purpose of this paper is two fold. In the following pages we prove common fixed point theorems for four mappings $A, B, S$ and $T$ (say) under the Meir-Keeler type $(\varepsilon, \delta$ ) condition, however, without imposing any additional condition on $\delta$ or using a $\phi$-contractive condition together with. Simultaneously we also show that none of the $A, B, S$ or $T$ is continuous at their common fixed point. Thus we not only generalize the Meir-Keeler type and Boyd-Wong type fixed point theorems, but also provide one more answer to the problem (see Rhoades [19]) on the existence of a contractive definition, which is strong enough to generate a fixed point but does not force the map to be continuous at the fixed point.
\end{abstract}

\section{Introduction}

It is known that while dealing with the common fixed point of four mappings, say $A$, $B, S$ and $T$ in a metric space $(X, d)$, the Meir-Keeler type $(\varepsilon, \delta)$ contractive condition of the type

(1) given $\varepsilon>0$ there exists a $\delta>0$ such that $\varepsilon \leq \max \{d(S x, T y), d(A x, S x), d(B y, T y),[d(A x, T y)+d(B y, S x)] / 2\}<\varepsilon+\delta$

$\Rightarrow d(A x, B y)<\varepsilon$

does not ensure the existence of a fixed point unless some additional condition is imposed on $\delta$ or a $\phi$-contractive condition of the form

(2) $d(A x, B y) \leq \phi(\max \{d(S x, T y), d(A x, S x), d(B y, T y),[d(A x, T y)+d(B y, S x)] / 2\})$

where $\phi: R_{+} \rightarrow R_{+}$is such that $\phi(t)<t$ for each $t>0$, is also used together with. To illustrate this argument we cite the following example:

Example 1. [13] Let $X=[0,2]$ and $d$ be the usual metric on $X$. Define $f: X \rightarrow X$ by

$$
f x=(1+x) / 2 \text { if } x<1, \quad f x=0 \text { if } x \geq 1
$$

Received September 5, 2002; revised March 18, 2004.

2000 Mathematics Subject Classification. 54H25.

Key words and phrases. Compatible maps, reciprocal continuity, common fixed point, contractive conditions. 
Then $f$ satisfies the contractive condition

$$
\varepsilon \leq \max \{d(x, y), d(x, f x), d(y, f y)\}<\varepsilon+\delta \Rightarrow d(f x, f y)<\varepsilon
$$

with $\delta(\varepsilon)=1$ for $\varepsilon \geq 1$ and $\delta(\varepsilon)=1-\varepsilon$ for $\varepsilon<1$ but $f$ does not have a fixed point.

In view of the above example it is inferred that the $(\varepsilon, \delta)$ contractive condition alone is not sufficient to ensure the existence of a common fixed point. In the known common fixed point theorems the existence of common fixed points is ensured by assuming either of the following conditions in addition to the $(\varepsilon, \delta)$ contractive condition:

(I) $\delta$ is nondecreasing (Pant $[10,11])$.

(II) $\delta$ is lower semicontinuous (Jungck [6], Jungck et al. [5]).

(III) Besides the $(\varepsilon, \delta)$ contractive condition the $\phi$-contractive condition is also assumed with or even without imposing any additional restriction on $\phi$ (Pant and Pant [12], Pant et al. [13]).

Jachymski [3, Proposition 4.2] has shown that the $(\varepsilon, \delta)$ contractive condition (1) implies the $\phi$-contractive condition (2) if $\delta$ is assumed nondecreasing. Pant et al. [13] has proved that the $(\varepsilon, \delta)$ contractive condition (1) implies the $\phi$-contractive condition (2) if $\delta$ is lower semicontinuous. A slightly different version of this result has been proved by Jachymski [3]. We thus see that if any of the condition (I) of (II) is imposed on $\delta$ then the $(\varepsilon, \delta)$ contractive condition (1) implies an analogous $\phi$-contractive condition and both the contractive conditions hold simulaneously alongwith (I) or (II).

In some results the contractive condition (1) has been replaced by a slightly weaker contractive condition of the form

(3) given $\varepsilon>0$ there exists a $\delta>0$ such that

$\varepsilon<\max \{d(S x, T y), d(A x, S x), d(B y, T y),[d(A x, T y)+d(B y, S x)] / 2\}<\varepsilon+\delta$

$\Rightarrow d(A x, B y) \leq \varepsilon$

Jachymski [3] has shown that contractive condition (1) implies (3) but (3) does not imply (1). The known common fixed theorems can be generalized if we assume the $(\varepsilon, \delta)$ contractive condition (3), however, without imposing any additional restriction on $\delta$ or taking the $\phi$-contractive condition together with. We do so in the present paper and prove a common fixed point theorem (Theorem 1) by assuming the contractive condition (3) together with the Lipschitz type analogue of a plane contractive condition; i.e., a condition of the form

$$
\begin{array}{r}
d(A x, B y)<\max \{d(S x, T y), k[d(A x, S x)+d(B y, T y)] / 2,[d(A x, T y)+d(B y, S x)] / 2\}, \\
0<k \leq 2
\end{array}
$$

however, without assuming any of the additional condition (I), (II) or (III). Theorem 2 slightly extends the result obtained in Theorem 1, whereas Theorem 3 is a special case of both the previous theorems. Simultaneously we also provide one more answer to the problem (see Rhoades [19]) on the existence of a contractive definition, which is strong enough to generate a fixed point but does not force the map to be continuous at the fixed point. For this we use the notion of Reciprocal Continuity. 
Two selfmappings $A$ and $S$ of a metric space $(X, d)$ are called reciprocally continuous (see Pant [15]) if $\lim _{n} A S x_{n}=A t$ and $\lim _{n} S A x_{n}=S t$ whenever $\left\{x_{n}\right\}$ is a sequence such that $\lim _{n} A x_{n}=\lim _{n} S x_{n}=t$ for some $t$ in $X$. If $A$ and $S$ both are continuous they are obviously reciprocally continuous. But, as shown and illustrated in the following pages, the converse is not true.

Two selfmaps $f$ and $g$ of a metric space $(X, d)$ are called compatible (see Jungck [6]) if $\lim _{n} d\left(f g x_{n}, g f x_{n}\right)=0$, whenever $\left\{x_{n}\right\}$ is a sequence in $X$ such that $\lim _{n} f x_{n}=$ $\lim _{n} g x_{n}=t$ for some $t$ in $X$.

Two selfmaps $f$ and $g$ of a metric space $(X, d)$ are called weakly compatible if they commute at their coincidence points.

The notion of compatible maps was introduced by Jungck [6] in 1986 by generalizing the concept of commutativity or say rather by generalizing the concept of weak commutativity. There are a number of other generalizations also of the concept of commutativity. It is, however, relevant to mention here that both commutativity and weak commutativity are independent of the notion of reciprocal continuity. We show in the following examples that if two maps are compatible or weakly compatible they are not necessarily reciprocally continuous and vice versa. by

Example 2. Let $X=[2,20]$ and $d$ be the usual metric on $X$. Define $f, g: X \rightarrow X$

$$
\begin{aligned}
& f x=6 \text { if } 2 \leq x<5, \quad f x=2 \quad \text { if } x \geq 5 \\
& g x=2 \text { if } 2 \leq x<5, \quad g x=x-3 \text { if } x \geq 5 .
\end{aligned}
$$

In this example $f 5=2=g 5$ but $g f 5=2, f g 5=6$. Thus $f$ and $g$ do not commute at their only coincidence point $x=5$. Let us now consider the sequence $\left\{x_{n}=5+1 / n: n>1\right\}$, then $\lim _{n} f x_{n}=2, \lim _{n} g x_{n} \rightarrow 2, \lim _{n} f g x_{n}=6=f 2$ and $\lim _{n} g f x_{n}=2=g 2$. Thus $f$ and $g$ are reciprocally continuous but are neither compatible nor weakly compatible.

Example 3. Let $X=[2,20]$ and $d$ be the usual metric on $X$. Define $f, g: X \rightarrow X$ by

$$
\begin{aligned}
& f x=6 \text { if } 2 \leq x \leq 5, \quad f x=2 \quad \text { if } x>5 \\
& g x=2 \text { if } 2 \leq x \leq 5, \quad g x=x-3 \text { if } x>5 .
\end{aligned}
$$

In this example $f$ and $g$ are reciprocally continuous but not compatible. To see this let us consider the sequence $\left\{x_{n}=5+1 / n: n>1\right\}$, then $\lim _{n} f x_{n}=2, \lim _{n} g x_{n} \rightarrow 2$, $\lim _{n} f g x_{n}=6=f 2$ and $\lim _{n} g f x_{n}=2=g 2$. Thus $f$ and $g$ are reciprocally continuous but not compatible. However, $f$ and $g$ are weakly compatible. by

Example 4. Let $X=[2,20]$ and $d$ be the usual metric on $X$. Define $f, g: X \rightarrow X$

$$
\begin{array}{llll}
f 2=2, f x=2 x+1 & \text { if } 2<x \leq 5, \quad f x=(x-1) / 2 & \text { if } x>5 \\
g 2=2, g x=(x+8) / 2 & \text { if } 2<x<5, \quad g x=x-3 & \text { if } x \geq 5 .
\end{array}
$$


Then $f$ and $g$ are compatible but not reciprocally continuous. To see this let us consider the sequence $\left\{x_{n}=5+1 / n: n>1\right\}$, then $\lim _{n} f x_{n} \rightarrow 2, \lim _{n} g x_{n} \rightarrow 2$, $\lim _{n} f g x_{n} \rightarrow 5 \neq f 2$ and $\lim _{n} g f x_{n} \rightarrow 5 \neq g 2$. For the sequence $\left\{x_{n}=2+1 / n: n>1\right\}$, $\lim _{n} f x_{n} \rightarrow 5, \lim _{n} g x_{n} \rightarrow 5, \lim _{n} f g x_{n} \rightarrow 2 \neq f 5$ and $\lim _{n} g f x_{n} \rightarrow 2=g 5$. Thus $f$ and $g$ are compatible but not reciprocally continuous.

\section{Results}

Theorem 1. Let $(A, S)$ and $(B, T)$ be compatible pairs of self mappings of a complete metric space $(X, d)$ such that

(i) $A X \subset T X, B X \subset S X$

(ii) given $\varepsilon>0$, there exists $\delta>0$ such that $\varepsilon<M(x, y)<\varepsilon+\delta \Rightarrow d(A x, B y) \leq \varepsilon ;$ where $M(x, y)=\max \{d(S x, T y), d(A x, S x), d(B y, T y),[d(A x, T y)+d(B y, S x)] / 2\}$

(iii) $d(A x, B y)<\max \{d(S x, T y), k[d(A x, S x)+d(B y, T y)] / 2,[d(A x, T y)+d(B y, S x)] / 2\}$, $0<k \leq 2$

Suppose that the mappings in one of the pairs $(A, S)$ or $(B, T)$ are reciprocally continuous. Then $A, B, S$ and $T$ have a unique common fixed point.

Proof. Let $x_{0}$ be any point in $X$. Define sequences $\left\{x_{n}\right\}$ and $\left\{y_{n}\right\}$ in $X$ given by the rule

$$
y_{2 n}=A x_{2 n}=T x_{2 n+1}, \quad y_{2 n+1}=B x_{2 n+1}=S x_{2 n+2} .
$$

This can be done by virtue of (i). Then by (ii) we get $d\left(y_{2 n}, y_{2 n+1}\right)<d\left(y_{2 n-1}, y_{2 n}\right)$. Similarly, $d\left(y_{2 n-1}, y_{2 n}\right)<d\left(y_{2 n-2}, y_{2 n-1}\right)$ and so on. Thus, $\left\{d\left(y_{n}, y_{n+1}\right)\right\}$ is a strictly decreasing sequence of positive numbers and, therefore, tends to a limit $r \geq 0$. If possible suppose $r>0$. Then given $\delta>0$ there exists a positive number $N$ such that for each $n \geq N$ we have

$$
r<d\left(y_{2 n}, y_{2 n+1}\right)=M(x, y)<r+\delta
$$

Selecting $\delta$ in (1) in accordance with (ii), for each $n \geq N$ we get $d\left(y_{2 n+2}, y_{2 n+1}\right)=$ $d\left(A_{1} x_{2 n+2}, A_{2} x_{2 n+1}\right)<r$. This, in turn, gives $d\left(y_{2 n+3}, y_{2 n+2}\right)<d\left(y_{2 n+1}, y_{2 n+2}\right)<r$, contradicting (1). Hence $\lim _{n \rightarrow \infty} d\left(y_{n}, y_{n+1}\right)=0$.

We now show that $\left\{y_{n}\right\}$ is a Cauchy sequence. Suppose it is not. Then there exists an $\varepsilon>0$ and a subsequence $\left\{y_{n_{i}}\right\}$ of $\left\{y_{n}\right\}$ such that $d\left(y_{n_{i}}, y_{n_{i+1}}\right)>2 \varepsilon$. Select $\delta$ in (ii) so that $0<\delta \leq \varepsilon$. Since $\lim _{n \rightarrow \infty} d\left(y_{n}, y_{n+1}\right)=0$, there exists an integer $N$ such that $d\left(y_{n}, y_{n+1}\right)<\delta / 6$ whenever $n \geq N$.

Let $n_{i} \geq N$. Then, there exist integers $m_{i}$ satisfying $n_{i}<m_{i}<n_{i+1}$ such that $d\left(y_{n_{i}}, y_{m_{i}}\right) \geq \varepsilon+(\delta / 3)$. If not, then

$$
\begin{aligned}
d\left(y_{n_{i}}, y_{n_{i+1}}\right) & \leq d\left(y_{n_{i}}, y_{n_{i+1}-1}\right)+d\left(y_{n_{i+1}-1}, y_{n_{i+1}}\right) \\
& <\varepsilon+(\delta / 3)+(\delta / 6)<2 \varepsilon
\end{aligned}
$$


a contradiction. Without loss of generality, we can assume $n_{i}$ to be odd. Let $m_{i}$ be the smallest even integer such that $d\left(y_{n_{i}}, y_{m_{i}}\right) \geq \varepsilon+(\delta / 3)$. Then $d\left(y_{n_{i}}, y_{m_{i}-2}\right)<\varepsilon+(\delta / 3)$ and

$$
\begin{aligned}
\varepsilon+(\delta / 3) & \leq d\left(y_{n_{i}}, y_{m_{i}}\right) \leq d\left(y_{n_{i}}, y_{m_{i}-2}\right)+d\left(y_{m_{i}-2}, y_{m_{i}-1}\right)+d\left(y_{m_{i}-1}, y_{m}\right) \\
& <\varepsilon+(\delta / 3)+(\delta / 6)+(\delta / 6)=\varepsilon+2(\delta / 3) .
\end{aligned}
$$

Also, $d\left(y_{n_{i}}, y_{m_{i}}\right) \leq M\left(x_{n_{i}+1}, x_{m_{i}+1}\right)<\varepsilon+2(\delta / 3)+(\delta / 6)<\varepsilon+\delta$,

that is, $\varepsilon+(\delta / 3) \leq M\left(x_{n_{i}+1}, x_{m_{i}+1}\right)<\varepsilon+\delta$. In view of (ii), this yields $d\left(y_{n_{i}+1}, y_{m_{i}+1}\right)<$ $\varepsilon$. But then

$$
\begin{aligned}
d\left(y_{n_{i}}, y_{m_{i}}\right) & \leq d\left(y_{n_{i}}, y_{n_{i}+1}\right)+d\left(y_{n_{i}+1}, y_{m_{i}+1}\right)+d\left(y_{m_{i}+1}, y_{m_{i}}\right) \\
& <(\delta / 6)+\varepsilon+(\delta / 6)=\varepsilon+(\delta / 3),
\end{aligned}
$$

which contradicts (2). Hence $\left\{y_{n}\right\}$ is a Cauchy sequence. Since $X$ is complete, there exists a point $z$ in $X$ such that $y_{n} \rightarrow z$. Also

$$
y_{2 n}=A x_{2 n}=T x_{2 n+1} \rightarrow z, \quad y_{2 n+1}=B x_{2 n+1}=S x_{2 n+2} \rightarrow z .
$$

Suppose that $A$ and $S$ are reciprocally continuous mappings. Then, reciprocal continuity of $A$ and $S$ implies that $A S x_{2 n} \rightarrow A z$ and $S A x_{2 n} \rightarrow S z$. Since $A$ and $S$ are compatible and $\lim _{n} S x_{2 n}=\lim _{n} A x_{2 n}=z$, we get $\lim _{n} d\left(A S x_{2 n}, S A x_{2 n}\right)=0$, that is, $d(A z, S z)=0$. Hence $A z=S z$. Since $A X \subset T X$, there exists a point $w$ in $X$ such that $A z=T w$. We claim that $T w=B w$. If $B w \neq T w$, using (iii) we get

$$
\begin{aligned}
d(A z, B w) & <\max \{d(S z, T w), k[d(A z, S z)+d(B w, T w)] / 2,[d(A z, T w)+d(B w, S z)] / 2\} \\
& =\max \{k[d(B w, T w)] / 2, d(B w, T w) / 2\}, \quad 0<k \leq 2 \\
& <d(B w, T w)=d(A z, B w),
\end{aligned}
$$

a contradiction. Hence $B w=T w$ and $S z=A z=T w=B w$. Since compatible maps commute at their coincidence points, we get $A S z=S A z$ and $B T w=T B w$. This further implies that $A A z=A S z=S A z=S S z$ and $B B w=B T w=T B w=T T w$. If $A z \neq A A z$, using (iii) we get

$$
\begin{aligned}
d(A z, A A z) & =d(B w, A A z)=d(A A z, B w) \\
< & \max \{d(S A z, T w), k[d(A A z, S A z)+d(B w, T w)] / 2, \\
& {[d(A A z, T w)+d(B w, S A z)] / 2\}) } \\
= & d(S A z, T w)=d(A A z, A z),
\end{aligned}
$$

a contradiction. Hence $A z=A A z=A S z$. Thus $A z$ is a common fixed point of $A$ and $S$. Similarly we obtain $B w(=A z)$ is a common fixed point of $B$ and $T$. Uniqueness of the common fixed point follows from (iii). The proof is similar when $B$ and $T$ are assumed compatible and reciprocally continuous. This completes the proof of the theorem.

Theorem 1 can be stated in different, albeit slightly improved version as follows.

Theorem 2. Let $(A, S)$ and $(B, T)$ be compatible pairs of self mappings of a complete metric space $(X, d)$ satisfying conditions (i) and (ii) of Theorem 1 above and 
(iii) $d(A x, B y)<\max \{d(S x, T y), k[d(A x, S x)+d(B y, T y)] / 2$,

$$
\left.k^{\prime}[d(A x, T y)+d(B y, S x)] / 2\right\}, \quad 0<k \leq 2, \quad 0<k^{\prime} \leq 1
$$

Suppose that the mappings in one of pairs $(A, S)$ or $(B, T)$ is reciprocally continuous. Then $A, B, S$ and $T$ have a unique common fixed point.

Proof. Let $x_{0}$ be any point in $X$. Define a sequence $\left\{y_{n}\right\}$ in $X$ as follows:

$$
y_{2 n}=A x_{2 n}=T x_{2 n+1}, \quad y_{2 n+1}=B x_{2 n+1}=S x_{2 n+2} .
$$

This can be done since $A X \subset T X$ and $B X \subset S X$. Following the steps as in Theorem 1 above it follows that $\left\{y_{n}\right\}$ is a Cauchy sequence. Since $X$ is complete, there exists a point $z$ in $X$ such that $y_{n} \rightarrow z$ as $n \rightarrow \infty$. Also,

$$
y_{2 n}=A x_{2 n}=T x_{2 n+1} \rightarrow z, \quad y_{2 n+1}=B x_{2 n+1}=S x_{2 n+2} \rightarrow z .
$$

Suppose that $A$ and $S$ are reciprocally continuous mappings. Then, reciprocal continuity of $A$ and $S$ implies that $A S x_{2 n} \rightarrow A z$ and $S A x_{2 n} \rightarrow S z$. Since $A$ and $S$ are compatible and $\lim _{n} S x_{2 n}=\lim _{n} A x_{2 n}=z$, we get $\lim _{n} d\left(A S x_{2 n}, S A x_{2 n}\right)=0$, that is, $d(A z, S z)=0$. Hence $A z=S z$. Since $A X \subset T X$, there exists a point $w$ in $X$ such that $A z=T w$. We claim that $T w=B w$. If $B w \neq T w$, using (iii) we get

$$
\begin{aligned}
d(A z, B w) & <\max \left\{d(S z, T w), k[d(A z, S z)+d(B w, T w)] / 2, k^{\prime}[d(A z, T w)+d(B w, S z)] / 2\right\} \\
& =\max \left\{k[d(B w, T w)] / 2, k^{\prime}[d(B w, T w)] / 2\right\} \\
& <d(B w, T w)=d(A z, B w),
\end{aligned}
$$

a contradiction. Hence $B w=T w$ and $S z=A z=T w=B w$. Since compatible maps commute at their coincidence points, we get $A S z=S A z$ and $B T w=T B w$. This further implies that $A A z=A S z=S A z=S S z$ and $B B w=B T w=T B w=T T w$. If $A z \neq A A z$, using (iii) we get

$$
\begin{aligned}
d(A z, A A z)= & d(B w, A A z)=d(A A z, B w) \\
< & \max \{d(S A z, T w), k[d(A A z, S A z)+d(B w, T w)] / 2, \\
& \left.\left.k^{\prime}[d(A A z, T w)+d(B w, S A z)] / 2\right\}\right) \\
= & d(S A z, T w)=d(A A z, A z),
\end{aligned}
$$

a contradiction. Hence $A z=A A z=A S z$. Thus $A z$ is a common fixed point of $A$ and $S$. Similarly we obtain $B w(=A z)$ is a common fixed point of $B$ and $T$. Uniqueness of the common fixed point follows from (iii). The proof is similar when $B$ and $T$ are assumed compatible and reciprocally continuous. This completes the proof of the theorem.

Remark. Setting $k=k^{\prime}=1$ in Theorem 2 above we get the following theorem.

Theorem 3. Let $(A, S)$ and $(B, T)$ be compatible pairs of self mappings of a complete metric space $(X, d)$ such that

(i) $A X \subset T X, B X \subset S X$ 
(ii) given $\varepsilon>0$, there exists $\delta>0$ such that

$\varepsilon<\max \{d(S x, T y), d(A x, S x), d(B y, T y),[d(A x, T y)+d(B y, S x)] / 2\}$

$<\varepsilon+\delta \Rightarrow d(A x, B y) \leq \varepsilon$

(iii) $d(A x, B y)<\max \{d(S x, T y),[d(A x, S x)+d(B y, T y)] / 2,[d(A x, T y)+d(B y, S x)] / 2\}$, Suppose that the mappings in one of pairs $(A, S)$ or $(B, T)$ is reciprocally continuous. Then $A, B, S$ and $T$ have a unique common fixed point.

We now give an example which illustrates the above theorems:

Example 5. Let $X=[2,20]$ and $d$ be the usual metric on $X$. Define $A, B, S$, $T: X \rightarrow X$ by

$$
\begin{aligned}
& A 2=2, \quad A x=3, \text { if } x>2, \\
& S 2=2, \quad S x=6, \text { if } x>2, \\
& B x=2, \text { if } x=2 \text { or } \geq 5, \quad B x=6, \text { if } 2<x<5, \\
& T 2=2, \quad T x=7+x, \text { if } 2<x<5, \quad T x=(x+1) / 2, \text { if } x \geq 5 .
\end{aligned}
$$

Then $A, B, S$ and $T$ satisfy all the conditions of above theorems and have a unique common fixed point $x=2$. It can be verified in this example that $A, B, S$ and $T$ satisfy contractive condition (ii) and (iii) of the above theorems and that $\delta(\varepsilon)=\varepsilon$ if $\varepsilon \geq 3$ and $\delta(\varepsilon)=3-\varepsilon$ if $\varepsilon<3$. We thus see that $\lim _{\varepsilon \rightarrow 3} \inf \delta(\varepsilon)=0 \neq \delta(3)$ and $\delta$ fails to be nondecreasing or lower semicontinuous. It can be verified that $A$ and $S$ are reciprocally continuous compatible mappings, however, all the mappings $A, B, S$ and $T$ are discontinuous at the common fixed point.

\section{Discussion}

1. The Meir-Keeler type $(\varepsilon, \delta)$ contractive condition alone does not guarantee the existence of a common fixed point unless some additional condition is imposed on $\delta$ (e.g. Jungck [6], Jungck et al. [5], Pant $[10,11]$ ) or a $\phi$-contractive condition has been used alongwith the $(\varepsilon, \delta)$ contractive condition (e.g. Pant and Pant [12], Pant et al. [13]. Jachymski [3] has shown that the $(\varepsilon, \delta)$ contractive condition (1) with nondecreasing $\delta$ implies a $\phi$-contractive condition. It is also true that the $(\varepsilon, \delta)$ contractive condition (1) with lower semicontinuous $\delta$ implies the $\phi$-contractive condition (e.g. see Pant et al. [13], also Jachymski [4]). Theorem 1 and 2 above have been proved without imposing any additional condition on $\delta$ and we have assumed a plane contractive condition instead of a $\phi$-contractive condition. Such plane contractive conditions, in general, do not guarantee the existence of common fixed points. Our theorems, thus improve the results known so far. Among the cases which can be obtained as a particular case of our theorems or which can be improved in the light of present theorems we mention due to Boyd and Wong [1], Carbone et al. [2], Jungck [6], Jungck et al. [5], Jachymski [3], Matkowski [8], Pant [14], Pant et al. [Theorem 1, [13]], Pant and Padaliya [Theorem 2, [16]], Park and Rhoades [18] and Singh and Kasahara [20]. 
2. Despite Lemma 2.2 of Jachymski [3], an $(\varepsilon, \delta)$ contractive condition does not imply the existence of common fixed point unless some additional condition is imposed on $\delta$. For example, Jungck [6] and Jungck et al. [5] assume $\delta$ to be lower semicontinuous. On the other hand Pant $[10,11]$ assume $\delta$ to be nondecreasing. In Theorem 1, 2 and 3 above, we have not imposed any additional condition on $\delta$. Example 2 illustrates our argument.

3 . In condition (iii) of Theorem 1 and 2 above $k$ can assume any value in the semiopen interval $(0,2]$ whereas $k^{\prime}$ can take any value in the interval $(0,1]$ in Theorem 2 . Thus our theorems generalize a number of similarly placed results by allowing $k$ and $k^{\prime}$ to take values other than 1.

4. It has been known since the paper of Kannan [7] in 1968 that there exist maps that have a discontinuity in their domain but which have fixed points. In 1988, Rhoades [19] posed an open problem - "Whether there exists a contractive definition which is strong enough to generate a fixed point, but which does not force the map to be continuous at the fixed point." The problem had remained open for more than one decade. Pant $[14,17]$ and Pant et al. [13] have provided some solutions to this problem. In the above theorems we have provided one more, however, a new type of answer to this problem. It may be observed that in the Example above none of the mappings is continuous at their common fixed point.

\section{Acknowledgement}

The authors are thankful to the referee for his valuable suggestions to improve the paper.

\section{References}

[1] D. W. Boyd and J. S. Wong, On nonlinear contractions, Proc. Amer. Math. Soc. 20(1969), 458-464.

[2] A. Carbone, B. E. Rhoades and S. P. Singh, A fixed point theorem for generalized contraction map, Indian J. Pure Appl. Math. 20(1989), 543-548.

[3] J. Jachymski, Common fixed point theorems for some families of maps, Indian J. Pure Appl. Math. 25(1994), 925-937.

[4] J. Jachymski, Equivalent conditions and Meir-Keeler type theorems, J. Math. Anal. Appl. 194(1995), 293-303.

[5] G. Jungck, K. B. Moon, S. Park and B. E. Rhoades, On generalizations of the Meir-Keeler type contraction maps: Corrections, J. Math. Anal. Appl. 180(1993), 221-222.

[6] J. Jungck, Compatible mappings and common fixed points, Internat. J. Math. Sci. 9(1986), 771-779.

[7] R. Kannan, Some results on fixed point, Bull. Cal. Math. Soc. 60(1986), 71-76.

[8] J. Matkowski, Integrable solutions of functional equations, Diss. Math. 127(1975).

[9] A. Meir and E. Keeler, A theorem on contraction mappings, J. Math. Anal. Appl. 28(1969), 326-329.

[10] R. P. Pant, Common fixed points of two pairs of commuting mappings, Indian J. Pure Appl. Math. 17(1986), 187-192. 
[11] R. P. Pant, Common fixed points of weakly commuting mappings, Math. Student 62(1993), 97-102.

[12] R. P. Pant and V. Pant, A unified fixed point theorem, Bull. Cal. Math. Soc. 91(1999), 227-232.

[13] R. P. Pant, P. C. Joshi and Vijay Gupta, A Meir-Keeler type fixed point theorem, Indian J. Pure Appl. Math. 32(2001), 1-9.

[14] R. P. Pant, On continuity at fixed points, J. Indian Math. Soc. (To Appear).

[15] R. P. Pant, A common fixed point theorem under a new condition, Indian J. Pure Appl. Math. 30(1999), 147-152.

[16] R. P. Pant and S. Padaliya, Reciprocal continuity and fixed point, Jñãnãbha 29(1999), 137143.

[17] R. P. Pant, A new common fixed point principle, Soochow J. Math. 27(2001), 287-297.

[18] S. Park and B. E. Rhoades, Extension of some fixed point theorems of Hegedus and Kasahara, Math. Seminar Notes 9(1981), 113-118.

[19] B. E. Rhoades, Contractive definitions and continuity, Contemporary Math. 72(1988), 233-245.

[20] S. L. Singh and S. Kasahara, On some recent results on common fixed points, Ind. J. Pure. Appl. Math. 13-17(1982), 757-761.

Department of Mathematics, Kumaon University, D.S.B. Campus, Nainital - 263 002, Inida.

Department of Mathematics, Kumaon University, D.S.B. Campus, Nainital - 263 002, Inida.

E-mail: vyomeshpant@yahoo.co.in

Department of Mathematics, Kumaon University Almora Campus, Almora, Uttaranchal, India. 